\title{
Diagnóstico e tratamento da intoxicação por chumbo em crianças e adultos
}

\section{Diagnosis and treatment of lead poisoning in children and adults}

\author{
Eduardo M. De Capitani
}

\begin{abstract}
RESUMO
O diagnóstico clínico da intoxicação por chumbo deve levar em conta os órgãos-alvo que determinam os sinais e sintomas mais característicos da intoxicação: cérebro, sistema hematopoiético, rins, e sistema nervoso periférico. O órgão alvo crítico é o cérebro, promovendo sinais e sintomas de encefalopatia mais ou menos acentuada, tais como, cefaléia, perda de memória, perda da concentração e atenção em tarefas corriqueiras, alterações de humor, com irritabilidade, depressão, insônia, ou sonolência, estupor, convulsões e coma, dependendo da dose e duração da exposição. O diagnóstico é confirmado pela dosagem de chumbo no sangue (plumbemia) ou na urina (plumbúria), ou de algum dos parâmetros de efeito do chumbo na cadeia de formação da hemoglobina, tais como o ácido deltaminolevulínico no sangue ou na urina e protoporfirina eritrocitária no sangue. $O$ tratamento da intoxicação é feito com quelantes. Por questão de custos e disponibilidade comercial, a experiência acumulada no Brasil restringe-se ao uso do dimercaprol (BAL), do versenato de cálcio (EDTACaNa2) e da D-penicilamina. Entretanto, os quelantes mais eficazes são o EDTACaNa2 por via parenteral e o ácido dimercapto succínico (DMSA) por via oral. O BAL pode ser utilizado juntamente com o EDTACaNa2 em casos de adultos com sintomatologia encefalopática, ou em crianças mesmo sem sintomas de SNC, pois diferentemente do EDTACaNa2 ele passa a barreira encefálica quelando chumbo presente no cérebro ou impedindo que chumbo mobilizado durante o uso do EDTA aja no cérebro da criança.
\end{abstract}

Palavras-chave: Chumbo. Intoxicação por Chumbo. Diagnóstico. Tratamento.

$\mathrm{P}$ ra fins de diagnóstico clínico da intoxicação por chumbo, deve-se ter em mente os órgãos-alvo que determinam os sinais e sintomas mais característicos da intoxicação: cérebro, sistema hematopoético, rins, sistema nervoso periférico.

\section{Sinais e sintomas}

Em praticamente todo tipo de exposição a chumbo, considerando a duração a curto ou longo prazos, em altas ou baixas concentrações, o órgão crítico alvo
Correspondência: Eduardo M. De Capitani Caixa Postal 7042 13076-970 - Campinas SP

Artigo recebido em 20/01/2009 Aprovado em 08/12/2009 
é sempre o cérebro, promovendo sinais e sintomas de encefalopatia mais ou menos acentuada, tais como, cefaléia, perda de memória, perda da concentração e atenção em tarefas corriqueiras, alterações de humor, com irritabilidade, depressão, insônia (ou sonolência excessiva). Esses sintomas podem progredir ou já de início manifestar-se de forma mais grave com alterações com distúrbios de comportamento evidentes (paranóia, delírios e alucinações), alterações da marcha e do equilíbrio (denotando já um comprometimento do cerebelo), agitação psicomotora e, em situações de exposição a concentrações muito elevadas em curto prazo, alterações de consciência como obnubilação, estupor e coma, precedidos, em alguns casos, de convulsões. A sequência de sintomas e sinais decorrentes da ação do metal sobre o SNC deve ser vista como possibilidades de ocorrência dependendo sempre da duração da exposição e da concentração de $\mathrm{Pb}$ no ambiente. Em crianças, pode-se verificar a ocorrência dos mesmos sinais e sintomas, sendo que os sinais mais intensos estão quase sempre relacionados à exposição por via digestiva (ingestão de solo contaminado, ou flocos de tinta de construções ou brinquedos deteriorados, com teores altos de chumbo, como nos EUA).

Convém lembrar que todos esses sinais e sintomas de encefalopatia são inespecíficos, nenhum deles de forma isolada, ou mesmo a soma deles, podem ser considerados como patognomônicos da intoxicação por chumbo inorgânico. A suspeita de que esse quadro clínico possa estar relacionado à intoxicação por chumbo surge com o conhecimento de que o paciente, adulto ou criança, tem algum tipo de exposição ao metal. A presença de outros sintomas, como cólica abdominal, sintomas gerais de fraqueza, fadiga, mialgia generalizada (ou mais localizada nas panturilhas), inapetência, queixas gástricas, perda da libido, associados a alterações em alguns parâmetros hematológicos, como diminuição nos níveis de hematócrito e hemoglobina, e presença de pontilhado basófilo nas hemácias, corroboram a hipótese diagnóstica.

\section{Exames laboratoriais e complementares}

A confirmação definitiva do diagnóstico se dá pela dosagem de algum parâmetro de dose interna, como chumbo no sangue (plumbemia) ou na urina (plumbúria), ou algum dos parâmetros de efeito do chumbo na cadeia de formação da hemoglobina, como aumento de ácido deltaminolevulínico na urina (ALA-U), aumento da dosagem de coproporfobilinogênio na urina
(CPU), aumento de protoporfirina IX, dosada na forma livre (EP) ou na forma zincada (ZPP), e diminuição na atividade da ácido deltaminolevulínico dehidratase (ALA-D). ${ }^{1,2}$

Nos quadros clínicos agudos suspeitos, em crianças, a radiografia simples de abdome pode ser útil na detecção de conteúdo radiopaco representativo de ingestão de material contendo chumbo. ${ }^{3}$ Em exposições crônicas a longo prazo, também em crianças, o efeito do chumbo no metabolismo dos ossos longos em fase de crescimento pode ser detectado como uma banda densa radiopaca na parte distal das metáfises, logo acima da cartilagem de conjugação, na zona de incorporação de tecido ósseo na metáfise. Esta imagem pode corroborar o diagnóstico de intoxicação crônica por $\mathrm{Pb}$. Essa alteração da imagem óssea parece estar relacionada com acúmulo de cálcio na região por distúrbio do metabolismo normal provocado pelo chumbo, e não ao depósito de chumbo, semelhante às linhas de sofrimento que marcam a ossatura de crianças durante estresses nutricionais, infecciosos ou metabólicos intensos durante a fase de crescimento. No entanto, em adultos, apesar do $\mathrm{Pb}$ ter grande afinidade pelos ossos e aí se acumular de forma praticamente definitiva (compartimento tecidual onde o $\mathrm{Pb}$ tem meia vida de eliminação ao redor de 30 anos), a quantidade de $\mathrm{Pb}$ incorporada pela matriz óssea não é suficiente para promover radiopacidade suficiente para ser detectada ao exame radiológico.

Pacientes com projéteis de arma de fogo alojados no corpo têm possibilidade de desenvolver sinais e sintomas de intoxicação por chumbo ao longo do tempo. Para que isso ocorra o projétil deve estar em contato com líquido sinovial ou líquido cefalorraquidiano. ${ }^{4}$ Existem na literatura inúmeros casos relatados de projéteis alojados em coxo-femurais e ombros, bem como em coluna vertebral em contato com o canal medular, produzindo elevação de plumbemia e sinais e sintomas crônicos de intoxicação por chumbo, em alguns casos com crises de agudização, dependendo de estresses metabólicos, nutricionais ou infecciosos. ${ }^{4}$

Atualmente existe técnica ainda não disponibilizada comercialmente, utilizando fluorescência de raios $\mathrm{X}$ (K-XRF), com ${ }^{57} \mathrm{Co}$ e ${ }^{109} \mathrm{Cd}$ como fontes de radiação, visando avaliar carga corpórea de $\mathrm{Pb}$ pela medida do metal acumulado nos ossos. A técnica usando ${ }^{57} \mathrm{Co}$ é aplicada à falange e a que utiliza ${ }^{109} \mathrm{Cd}$ é aplicada à tíbia ou calcâneo. Essas técnicas foram idealizadas e utilizadas em estudos de cinética do $\mathrm{Pb}$ em ossos e posteriormente na avaliação de carga corpórea 
em expostos. ${ }^{5,6}$ Esse exame serve para auxiliar no diagnóstico e na indicação e seguimento de tratamento quelante da intoxicação crônica por $\mathrm{Pb}^{7-9}$ As medidas feitas a partir da crista da tíbia e de falanges apresentam boa correlação com PbU-EDTA pós teste de mobilização de chumbo (TMC) e com o conteúdo de Pb em biópsia. ${ }^{9}$

A interpretação das dosagens sanguíneas e urinárias de $\mathrm{Pb}$, e de porfirinas, como de qualquer outro exame laboratorial bioquímico, depende da comparação com valores de referência (VR) obtidos de populações equivalentes, tidas como sadias e não expostas, e de valores limites de tolerância biológica, chamados de índices biológicos de exposição (IBE).

Os IBE são definidos para monitorização de trabalhadores expostos. São valores que devem ter correspondência ou correlação com valores limites da substância no ar do ambiente de trabalho, que por sua vez são chamados de Limites de Tolerância Ambientais (LTA). Nesse sentido, duas situações referentes ao diagnóstico de intoxicação devem ser tratadas separadamente: i) exposição ocupacional, para a qual existem valores limites (IBE) para dosagens de indicadores biológicos de exposição. Dosagens de indicadores abaixo do IBE apontam para baixa probabilidade de ocorrência de intoxicação clínica. Os valores de IBE são sempre superiores aos valores de referência (VR) para a população não exposta; e ii) exposição ambiental, de adultos e crianças, na qual existirão valores limites para diferentes faixas etárias de crianças, e valores de referência (VR), que podem ser regionais ou nacionais.

Nas duas situações, o critério diagnóstico de intoxicação clínica por chumbo deve, necessariamente, incluir a positividade das três ocorrências: a) presença de sinais e sintomas compatíveis com intoxicação; b) comprovação de exposição ocupacional ou ambiental a $\mathrm{Pb}$, com duração e intensidade compatíveis com os sinais e sintomas; c) dosagens aumentadas de $\mathrm{Pb}$ no sangue e/ou porfirinas no sangue ou na urina, comparadas com valores de referência ou limites de tolerância biológica (IBE). A positividade de apenas um ou dois desses fatores não autoriza o diagnóstico de intoxicação, sendo necessária a investigação mais cuidadosa visando preencher os três critérios. (Quadros 1 e 2). Os IBEs contidos na Norma Regulamentadora $\mathrm{N}^{\circ} 7$ do Ministério do Trabalho não refletem o conhecimento científico atual com relação à probabilidade de ocorrência de efeitos adversos crônicos irreversíveis, como a neuropatia periférica de membros, em níveis de exposição considerados seguros pela legislação, necessitando mudança urgente.

Para crianças expostas ambientalmente, a maior experiência clínica e epidemiológica acumulada no mundo é a dos norte-americanos, em função do problema crônico e grave de contaminação de tintas usadas nas décadas de 40, 50 e 60 em residências, e que hoje, devido à deterioração e contaminação dos espaços intra e peri-domiciliar, são fonte perene de exposição de crianças que habitam esses locais. Baseado nessa experiência de avaliação de risco epidemiológico e manejo de milhares de casos clínicos de intoxicação, o Centre for Disease Control and Prevention, dos EUA, publicou recomendações sobre o manejo de crianças em áreas contaminadas, lastreado em sintomatologia, sinais clínicos e, basicamente, em níveis de plumbemia medidos regular e periodicamente, recomendações estas resumidas na Quadro 3. ${ }^{10,11}$

\section{Teste de Mobilização de Chumbo (TMC)}

Mesmo apresentando níveis de chumbo no sangue acima de $10 \mu \mathrm{g} / \mathrm{dL}$, apenas têm indicação formal de tratamento quelante as crianças que mostrem níveis acima de $44 \mu \mathrm{g} / \mathrm{dL}$, em função da altíssima probabilidade de desencadeamento de sintomas e lesões graves de SNC de caráter subagudo e crônico nesses níveis de plumbemia. ${ }^{10,11}$ Crianças mostrando níveis séricos de $\mathrm{Pb}$ entre 20 e $44 \mu \mathrm{g} / \mathrm{d}$, inquietam o pediatra e os pais, pois se sabe que tais níveis podem estar associados a alterações comportamentais e cognitivas crônicas. ${ }^{12,13}$ Alguns estudos, no entanto, têm mostrado que a redução desses níveis, através de tratamento quelante ou ao longo do tempo sem tratamento algum, para níveis próximos dos valores de referência, não resulta em melhora dos índices nos testes de cognição em crianças. ${ }^{14-17}$ Por outro lado, efeitos adversos neurológicos centrais e periféricos foram detectados em um grupo de 281 adultos jovens com história de exposição a chumbo ambiental na infância com dosagens médias à época de 49,3 $\mu \mathrm{g} / \mathrm{dl}, 20$ anos antes e não tratados, comparados com 287 controles não expostos. ${ }^{16}$ Apesar da não indicação de tratamento imediato dessas crianças, há necessidade de avaliação complementar quanto à carga corpórea de chumbo localizada em compartimentos de meia vida intermediária passíveis de serem mobilizados e causar dano. Essa avaliação é feita através do teste de mobilização de chumbo com uso de dose padronizada de droga quelante. 


\section{Quadro 1}

Sinais, sintomas e alterações laboratoriais e de exames complementares nos quadros suspeitos de intoxicação aguda/subaguda e crônica por chumbo inorgânico

\begin{tabular}{ll} 
Indicadores & Intoxicação Aguda/Subaguda \\
\hline Sinais e Sintomas & Cólicas abdominais que não respondem a \\
& antiespasmódicos; anorexia; palidez da \\
& pele; icterícia (nos casos com hemólise); \\
& náuseas; vômitos; constipação; agitação \\
& psicomotora; irritabilidade; ataxia; dese- \\
& quilíbrio; obnubilação; estupor; convul- \\
& sões; coma; sinais de insuficiência renal \\
& aguda; hipertensão arterial transitória
\end{tabular}

Alterações laborato-
riais que corrobo-
ram suspeita clíni-
ca, definindo o diag-
nóstico

- $\mathrm{Hb}<10 \mathrm{mcg} / \mathrm{dl}$

- Ponteado basófilo nas hemácias

- ALA-U elevado

- CPU elevado

- EP (ou ZPP) elevados

- Radiografia de abdome de crianças mostrando material radiopaco no TGI

- História de exposição ocupacional ou ambiental compatível (duração e intensidade)

- $\mathrm{PbS}>25 \mu \mathrm{g} / \mathrm{dl}$ em crianças

- $\mathrm{PbS}>60 \mu \mathrm{g} / \mathrm{dl}$ em adultos *

\section{Intoxicação Crônica}

Queixas inespecíficas como fadiga, palidez; perda de memória, irritabilidade, alterações de humor, perda da libido; anorexia leve, mialgia generalizada (ou mais localizada nas panturrilhas); queimação epigástrica; parestesias e perda de força muscular nas extremidades; sinais de insuficiência renal; hipertensão arterial; linhas de deposição de sulfeto de chumbo nas gengivas (linhas de Burton)

- $\mathrm{Hb}<10 \mathrm{mcg} / \mathrm{dl}$

- Ponteado basófilo nas hemácias

- ALA-U elevado

- CPU elevado

- EP (ou ZPP) elevados

- Radiografia de ossos longos em crianças (punho ou tíbia, na área de conjugação) mostrando bandas densas.

- História de exposição ocupacional ou ambiental compatível (duração e intensidade)

- PbS entre 25 e $44 \mu \mathrm{g} / \mathrm{dl}$ em crianças, fazer TMC

- $\mathrm{PbS}>40 \mu \mathrm{g} / \mathrm{dl}$ em adultos afastados da exposição ocupacional

- $\mathrm{PbS}>60 \mu \mathrm{g} / \mathrm{dl}$ em adultos expostos

Fonte: Nadig, 1994 (modificado) ${ }^{3}$

* Esse valor é uma referência apenas, sendo que em quadros agudos exuberantes em termos de sinais e sintomas, podem ocorrer níveis de $\mathrm{PbS}$ menos elevados, abaixo de $60 \mu \mathrm{g} / \mathrm{dl}$, o que não descarta o diagnóstico de intoxicação merecendo tratamento específico com agentes quelantes.

\section{Quadro 2}

Ações de controle desencadeadas conforme níveis dos indicadores biológicos para adultos

\begin{tabular}{ll}
$\begin{array}{l}\text { Situação dos parâmetros laboratoriais } \\
\text { (indicadores de dose e efeito) }\end{array}$ & Ações necessárias \\
\hline $\mathrm{PbS}<40 \mu \mathrm{g} / \mathrm{dL}$ & $\bullet$ Monitorização regular e periódica \\
$\mathrm{ALA}-\mathrm{U}<4,5 \mathrm{mg} / \mathrm{g} \mathrm{C}$ & \\
$\mathrm{ZPP}<40 \mu \mathrm{g} / \mathrm{dL}$ & $\bullet$ monitorização regular e periódica \\
\hline $\mathrm{PbS}$ entre 40 e $60 \mu \mathrm{g} / \mathrm{dl}$ & $\bullet$ avaliação médica periódica de sintomas \\
$\mathrm{ALA}-\mathrm{U}$ entre $4,5 \mathrm{mg} / \mathrm{gC}$ e $10 \mathrm{mg} / \mathrm{gC}$ & $\begin{array}{l}\text { afastamento da exposição no caso de sintomas } \\
\text { exuberantes de intoxicação e reavaliação laboratorial } \\
\mathrm{ZPP} \text { entre } 40 \text { e } 100 \mu \mathrm{g} / \mathrm{dL}\end{array}$ \\
& $\begin{array}{l}\text { em seguida } \\
\end{array}$
\end{tabular}

$\mathrm{PbS}>60 \mu \mathrm{g} / \mathrm{dl}$

ALA-U $>10 \mathrm{mg} / \mathrm{gC}$

$\mathrm{ZPP}>100 \mu \mathrm{g} / \mathrm{dL}$
- afastamento da exposição **

- avaliação clínica criteriosa

- tratamento se necessário

* basta um dos três resultados estar alterado para definir a ação no campo direito do quadro** o retorno ao trabalho só poderá ser efetivado com valores dos parâmetros dosados abaixo do VR · 


\section{Quadro 3}

Níveis de plumbemia em crianças em áreas de exposição ambiental a chumbo e recomendações de ações específicas, ambientais, de avaliação clínica e de tratamento, para cada nível

\begin{tabular}{|c|c|}
\hline Plumbemia (mcg/dl) & Intervenções Necessárias (ambientais, de avaliação clínica e terapêutica) \\
\hline \multirow[t]{3}{*}{$<\mathrm{ou}=9$} & - baixo risco de ocorrência de sinais e sintomas de intoxicação \\
\hline & - valores próximos dos VRs para populações não expostas \\
\hline & - reavaliação a cada 6 m ou um ano, dependendo da faixa etária \\
\hline \multirow[t]{3}{*}{$10-14$} & - baixo risco de ocorrência de sinais e sintomas de intoxicação \\
\hline & - reavaliação a cada $6 \mathrm{~m}$ ou um ano, dependendo da faixa etária \\
\hline & $\begin{array}{l}\text { - no caso de número significativo de crianças estar nessa faixa de resultado, a área deve ser } \\
\text { considerada quanto à avaliação de contaminação ambiental e possível remediação }\end{array}$ \\
\hline \multirow[t]{4}{*}{$15-19$} & - baixo risco de sinais e sintomas de intoxicação \\
\hline & - reavaliação a cada 6 m ou um ano, dependendo da faixa etária \\
\hline & - avaliação de possíveis fontes de exposição intradomiciliar, hábito alimentar. \\
\hline & - fazer hemograma e dosar ferro sérico \\
\hline \multirow[t]{6}{*}{$20-44$} & - risco moderado / alto de sinais e sintomas de intoxicação conforme a faixa etária \\
\hline & $\begin{array}{l}\text { - probabilidade de ocorrência de distúrbios de aprendizado em situação de exposição a esses } \\
\text { níveis por longos períodos }\end{array}$ \\
\hline & - necessária avaliação clínica criteriosa (incluindo neurológica) \\
\hline & - afastamento da exposição \\
\hline & - medidas de controle da fonte de exposição e remediação ambiental \\
\hline & - nos casos de PbS entre 25 e 44 mcg/dl está indicado TMC (Teste de Mobilização de Chumbo) \\
\hline \multirow[t]{5}{*}{$45-69$} & - risco altíssimo de ocorrerem sinais e sintomas de intoxicação \\
\hline & - avaliação clínica criteriosa e especializada \\
\hline & - afastamento imediato da exposição \\
\hline & - tratamento específico com agente quelante (EDTACaNa ${ }_{2}+\mathrm{BAL}$, ou DMSA via oral) \\
\hline & - controle da fonte de exposição e remediação ambiental. \\
\hline \multirow[t]{2}{*}{$>\mathrm{ou}=70$} & - tratamento específico com agentes quelantes de imediato \\
\hline & - todas as outras medidas de afastamento da exposição e controle ambiental \\
\hline
\end{tabular}

Fonte: CDCP $(1991)^{10}$

O TMC tem por objetivo clínico estimar a carga corpórea de $\mathrm{Pb}$, através da medida da quantidade de chumbo excretada na urina (plumbúria ou PbU), a partir da administração de uma dose única padrão de quelante. ${ }^{17}$ Tradicionalmente esse teste (também chamado de Teste de Provocação) é feito usando-se o versenato de cálcio $\left(\mathrm{EDTACaNa}_{2}\right)$, mas pode ser re- alizado com D-penicilamina e, eventualmente, com DMSA. ${ }^{17}$

A interpretação básica do TMC é de que a plumbúria produzida após administração do quelante, reflita a carga de chumbo presente em compartimentos mobilizáveis, e provavelmente não a carga corpórea total de chumbo, presente em tecidos de meia-vida 
prolongada, como ossos compactos, dentina, e rins. ${ }^{9,}{ }^{17} \mathrm{~A}$ importância clínica do teste está na possibilidade de avaliar a necessidade de tratamento quelante, em ciclos repetidos, para o chumbo localizado em grande quantidade em compartimentos mobilizáveis, tais como, fígado, baço, vísceras ocas, eritrócitos, cérebro e, provavelmente, osso trabecular, depósitos estes que podem representar risco de ocorrência de quadros clínicos graves quando mobilizados repentinamente. Resultados de PbU após dose teste de EDTA (PbUEDTA) têm boa correlação com níveis de plumbemia, principalmente quando a exposição se deu recentemente. Para exposições no passado a correlação não é tão boa. ${ }^{17}$

O TMC é útil na indicação ou não de tratamento em casos clínicos nos quais a sintomatologia é leve, ou os níveis de $\mathrm{PbS}$ estão baixos, em situação de exposição considerada prolongada a níveis de concentração moderados ou altos. O teste nunca deve ser realizado em pacientes com sintomatologia exuberante e típica de intoxicação por chumbo, ou com níveis de $\mathrm{PbS}$ acima de $100 \mathrm{mcg} / \mathrm{dl}$, pois a dose padrão do teste é baixa e pode desencadear mobilização de $\mathrm{Pb}$ de compartimentos teciduais em quantidade grande, $\mathrm{o}$ suficiente para produzir piora de sintomas. ${ }^{17}$

O TMC é realizado em adultos usando-se EDTACaNa2 em dose média de $25 \mathrm{mg} / \mathrm{kg}$ (cerca de $1 \mathrm{a} 2 \mathrm{~g}$ ) por via endovenosa, em infusão de 1 a 2 horas de duração (em 300 a 500ml de SF 0,9\% ou SG 5\%), seguida de coleta de urina de 24 horas para dosagem da plumbúria (PbU-EDTA - 24horas). Para resultados de PbU-EDTA-24hs acima de $600 \mu \mathrm{g}$, a interpretação é de que existe carga corpórea mobilizável de chumbo, que pode ou não necessitar de tratamento quelante. $\mathrm{O}$ tratamento quelante estará indicado formalmente quando o PbU-EDTA-24hs resultar acima de 1000-1500 $\mu \mathrm{g}$. Os pacientes com resultados entre 600 e 1000-1500 $\mu \mathrm{g}$ não necessariamente precisam usar quelantes, podendo se beneficiar apenas do afastamento da exposição por tempo mais prolongado, quando o organismo terá tempo de excretar o excesso de $\mathrm{Pb}$ pelo rim.

O TMC está indicado para crianças com níveis de $\mathrm{PbS}$ entre 25 e $44 \mu \mathrm{g} / \mathrm{dl}$, desde que afastadas da exposição ambiental. Está contra-indicado em crianças com níveis de $\mathrm{PbS}$ acima de $45 \mu \mathrm{g} / \mathrm{dl}$. A dose preconizada é de $500 \mathrm{mg} / \mathrm{m}^{2}$ em SG 5\%, em infusão de 1 hora. ${ }^{10} \mathrm{Um}$ TMC em criança será considerado positivo se o índice PbU-EDTA24horas/dose EDTACaNa em $\mathrm{mg}$ for maior que $0,6{ }^{18}$
Pode-se realizar TMC com D-penicilamina, na dose de 450 a 500mg em dose única oral, para adultos, dada à noite e dosando $\mathrm{PbU}$ em urina das 8 horas seguintes. O CDCP (1991) não indica uso de D-penicilamina para TMC em crianças. $\mathrm{O}$ valor de corte para indicação de tratamento quelante é de $300 \mu \mathrm{g} .{ }^{9}, 17$

O DMSA pode também ser usado como quelante na realização de TMC, na dosagem de $10 \mathrm{mg} / \mathrm{kg}$ em administração oral única, porém ainda não existe padronização da interpretação dos resultados com esse quelante. Estudo de $1995^{19}$ mostrou que o PbU após provocação com DMSA é, em média, menor quando comparado com dose equivalente de versenato de cálcio. ${ }^{19}$

Um dos incômodos na realização do TMC é a necessidade de coleta de urina de 24 horas para a plumbúria. Nesse sentido, algumas propostas alternativas têm sido feitas na literatura, com testes sendo avaliados com urina de 3,6 ou 8 horas após administração da droga. ${ }^{9}{ }^{918,20,21}$ Aparentemente, os três períodos de coleta fornecem resultados de PbU-EDTA com boa correlação com resultados de 24 horas, indicando boa segurança na substituição dos períodos de tempo na realização dos TMC. Os coeficientes de correlação obtidos nesses trabalhos foram: $r=0,86$ para coleta de 3 horas e $r=0,98$ para coleta de 6 horas.

Apesar de controverso em algumas situações clínicas, quanto à interpretação de resultados, o TMC é uma ferramenta importantíssima na avaliação clínica de adultos e crianças expostos a chumbo, visando avaliar carga corpórea mobilizável. As seguintes vantagens podem ser enumeradas: a) avalia indiretamente a carga mobilizável de $\mathrm{Pb}$ no organismo de expostos; b) auxilia na programação da dose e duração dos ciclos de tratamento quelante quando este for indicado; c) avalia a presença de carga corpórea anormal em exposições passadas; d) auxilia na decisão sobre se manifestações clínicas inespecíficas em paciente exposto a $\mathrm{Pb}$ no passado podem ser atribuídas à carga corpórea mobilizável atualmente. ${ }^{17}$

\section{Tratamento quelante da intoxicação}

O termo "quelação" provém do inglês "chelation", que por sua vez vem do grego, a partir de khélê, que significa pinça ou mandíbula. O latim também fornece "chêle", derivado do grego, significando "os braços da constelação de escorpião". O sentido químico do termo está ligado à ação de pinçar, agarrar, de transformar uma substância (o quelante) e um metal qualquer 
num complexo químico estável através de uma ligação covalente.

Os agentes quelantes são relativamente inespecíficos, e sua eficácia depende da afinidade química pelo metal causador da intoxicação; das características toxicocinéticas (coeficiente de partição; passagem por membranas; passagem por barreiras hematoencefálica e placentária, por exemplo); e da capacidade de excreção do complexo quelado. O quelante ideal deve ter alta hidrossolubilidade (para garantir máxima excreção urinária do quelato); não sofrer biotransformação significativa; ter boa distribuição através dos tecidos orgânicos, onde o metal tóxico possa estar depositado ou agindo; ter especificidade de ligação com o metal em questão e pouca ou nenhuma afinidade com metais essenciais como zinco, ferro, cobre, magnésio, manganês; ter pouca afinidade com o cálcio, evitando produzir hipocalcemia clínica; ter capacidade química de formar um quelato estável no $\mathrm{pH}$ dos diversos compartimentos corpóreos; ter boa taxa de excreção renal (ou eventualmente, hepatobiliar); e ter baixo índice de efeitos colaterais nas doses terapêuticas. ${ }^{22,23}$

Os quelantes disponíveis para tratamento da intoxicação pelo chumbo encontram-se listados no Quadro 4. Por questões de custos e disponibilidade comercial, a experiência acumulada no Brasil restringe-se ao uso do dimercaprol, do versenato de cálcio e, menos, da D-penicilamina.

Os quelantes mais eficazes na intoxicação por chumbo são o EDTACaNa ${ }_{2}$ por via parenteral e o DMSA por via oral. O BAL (dimercaprol) pode ser utilizado juntamente com o EDTACaNa $\mathrm{em}_{2}$ casos de adultos com sintomatologia encefalopática ou em crianças mesmo sem sintomas de SNC, pois diferentemente do EDTACaNa ${ }_{2}$ ele passa a barreira encefálica quelando chumbo presente no cérebro ou impedindo que chumbo mobilizado durante o uso do EDTA aja no cérebro da criança. O DMPS e a Dpenicilamina seriam os quelantes menos indicados devido à baixa eficácia e potenciais efeitos colaterais.

O EDTACaNa ${ }_{2}$ é um ácido da família dos ácidos poliaminocarboxílicos com afinidade química por diversos metais. Tem sido usado há várias décadas como reagente industrial e em processos de química analítica por sua capacidade de quelar cátions divalentes e trivalentes. ${ }^{23}$ Foi introduzido na farmacopéia como quelante em casos de intoxicação por chumbo na década de 50 e é usado para esse fim desde então. A molécula original, sódica, sem cálcio em sua estrutura, tem também afinidade por vários metais, incluindo o cálcio, e seu uso pode eventualmente causar hipocalcemia. No entanto, pode ser usado na intoxicação pelo chumbo desde que cuidados com a calcemia incluindo reposição de $\mathrm{Ca}$, sejam tomados periodicamente. A molécula do versenato de cálcio não apresenta esse problema potencial, pois a ligação do metal é feita pelo deslocamento do cálcio que, liberado, não chega a causar efeitos.

Trata-se de molécula altamente hidrossolúvel, com um volume de distribuição praticamente igual ao volume do compartimento extracelular. Devido à sua pouca ou nenhuma lipossolubilidade (é altamente polar, apresentando-se sempre na forma iônica), o versenato de cálcio atravessa mal as barreiras hematoencefálica e placentária. Por suas características de hidros-

\section{Quadro 4}

Quelantes usados na prática clínica das intoxicações por chumbo

Agente quelante (nome científico) e [nome comercial]

Dimercaprol (BAL) ou (2,3-dimercaptopropanol)

ácido dimercapto propanil-1- sulfônico (DMPS)

$\left[\right.$ Dimaval $^{\circledR} ;$ Unithiol $^{\circledR}$ ]

ácido dimercaptosuccínico (DMSA) [Chemet ${ }^{\circledR} ;$ Succimer $\left.^{\circledR}\right]$

D-penicilamina [cobreprimine ${ }^{\circledR}$; Depen $^{\circledR}$ ]
Outros metais quelados e outros usos terapêuticos

arsênio, mercúrio, chumbo, ouro

arsênio, mercúrio, chumbo

chumbo, arsênio, mercúrio, alumínio

cobre, chumbo, arsênio, mercúrio, ouro

doença de Wilson, cirrose biliar

ácido etilenodiamino- tetracético cálcico dissódico

$\left(\mathrm{EDTACaNa}_{2}\right)$ [Versenato de calcio]

chumbo 
solubilidade e polaridade, menos de 5\% da dose administrada por via oral são absorvidos, não sendo recomendada essa via para uso terapêutico. Existem evidências de que o chumbo tenha sua absorção aumentada no trato gastrintestinal pela complexação com o EDTA.

Estima-se que menos de 5\% da dose administrada endovenosamente chegue ao líquor. A taxa de eliminação renal é aproximadamente a mesma da filtração glomerular, correlacionando-se bem com o clearance (depuração) de creatinina. Pode ser administrado pela via intramuscular, com boa absorção e boa eficácia, mas a dor local limita o número de injeções possíveis e constitui seu único inconveniente. ${ }^{24-26}$

A administração parenteral, tanto endovenosa quanto intramuscular, promove a melhoria clínica da intoxicação por chumbo, em poucas horas. Há redução dos níveis plasmáticos de chumbo em alguns dias e reversão dos efeitos hematológicos, como restabelecimento dos níveis normais de ALA-D, ALA-U e protoporfirina eritrocitária (esta se normaliza com mais vagar, pois sendo eritrocitária depende da meia-vida da hemácia, que é de 120 dias), e aumento da excreção urinária de chumbo em dezenas a centenas de vezes o valor pré-quelação. ${ }^{17,23}$

O efeito tóxico mais temido do versenato (tanto o sódico quanto o cálcico), mas pouco frequente, está relacionado a um possível dano renal. Doses altas repetidas (mais de $75 \mathrm{mg} / \mathrm{kg} / \mathrm{dia}$, por mais de 10 dias) podem causar vacuolização hidrópica das células tubulares proximais, com perda da borda em escova e degeneração celular. Alterações nos túbulos distais são mais raras. Apesar de dramático, esse efeito é, em geral, dose-dependente e reversível com a interrupção do tratamento. Utilizando-se doses habituais entre 15 e $25 \mathrm{mg} / \mathrm{kg} /$ dia não há relatos de danos renais significativos.

O outro grupo de efeitos colaterais do uso do versenato está relacionado ao fato dele quelar somente o chumbo contido no compartimento extracelular (plasma e líquido extra vascular), criando assim um gradiente de concentração entre esse compartimento e outros diversos compartimentos cinéticos por onde o chumbo se distribui desigualmente. Isso pode provocar uma mobilização (redistribuição) muito rápida do chumbo desses compartimentos em direção ao plasma, causando elevação abrupta de plumbemia que será redistribuída, podendo causar sintomas encefálicos, e piora dos sintomas já existentes, como cólica abdominal. ${ }^{27} \mathrm{~A}$ administração de BAL por via intramuscular a cada 4 horas, que passa a barreira hematoencefáli- ca, está indicada nessas situações, visando conter a ação do chumbo no cérebro.

A dose regular de versenato para uso endovenoso varia de 15 a $25 \mathrm{mg} / \mathrm{kg} / \mathrm{dia}$, podendo alcançar 50 a $75 \mathrm{mg} / \mathrm{kg} / \mathrm{dia}$, dependendo dos níveis de plumbemia e gravidade da sintomatologia e dos sinais clínicos. A dose deve ser administrada em soro fisiológico a $0,9 \%$ ou soro glicosado a $5 \%$, em volume que não ultrapasse $500 \mathrm{~mL}$, em infusão gotejante com duração mínima de 1 h 30 min. a 2 h. Durações de infusão maiores (até cerca de 3h) são mais seguras e garantem eficácia semelhante de tratamento. Hidratação adequada que garanta boa diurese é medida essencial e precede qualquer outro procedimento terapêutico. A administração é feita em ciclos de 3 a 5 dias, com intervalos entre ciclos de no mínimo 2 dias, visando dar tempo para re-equilíbrio entre os compartimentos.

A eficácia do tratamento é avaliada pela remissão dos sintomas e pela dosagem de chumbo excretado na forma quelada durante cada $24 \mathrm{~h}$ de tratamento. A repetição dos ciclos de 3 a 5 dias dependerá dos resultados dessas dosagens urinárias (plumbúria de 24 h) durante cada ciclo, e dos níveis de plumbemia verificados nos intervalos dos ciclos. A medida de plumbemia durante os dias de tratamento não é merecedora de crédito, pois vai refletir o processo de mobilização de chumbo dos diversos compartimentos em direção ao plasma e não refletirá o estado de equilíbrio entre eles.

O DMSA é um derivado hidrossolúvel, análogo do dimercaprol (BAL). Foi sintetizado em 1940, mas foi utilizado como quelante de chumbo e mercúrio em casos de intoxicação apenas em 1965, na China. Durante as décadas de 70 e 80 foi utilizado com certa frequência na Ásia e Europa, até ser aprovado pela Food and Drug Admnistration (FDA, EUA), em 1991, para tratamento de crianças sintomáticas por exposição a chumbo, com níveis de plumbemia acima de $45 \mathrm{mcg} / \mathrm{dL} .{ }^{28}$

Embora quimicamente similar ao dimercaprol, a molécula do DMSA contém dois ácidos carboxílicos, além dos dois grupamentos sulfidrila (-SH), que modificam a cinética de distribuição e seu espectro de afinidade pelos metais. DMSA é absorvido pelo trato gastrintestinal de forma incompleta, mas rápida. Estima-se que a taxa de absorção seja no máximo de $20 \%$. Pico plasmático ocorre em cerca de $1 \mathrm{a}$ duas horas após administração oral e o quelante tem volume de distribuição pequeno, permanecendo a maior parte da dose no compartimento plasmático. No plasma, $95 \%$ encontram-se ligados à albumina. O DMSA sofre 
biotransformação, provavelmente hepática, sendo excretado na forma de dissulfetos mistos DMSA-cisteína em $90 \%$ da dose administrada, e os restantes $10 \%$ inalterados. Foi demonstrado haver ciclo enterohepático do agente. A meia-vida de eliminação urinária do DMSA é de aproximadamente $48 \mathrm{~h}$. O pico de excreção do produto de biotransformação em DMSAcisteína ocorre em $4 \mathrm{~h}$ após ingestão. A DL50 é de cerca de $5 \mathrm{~g} / \mathrm{kg}$, contra $1010 \mathrm{mg} / \mathrm{kg}$ para o BAL e $1 \mathrm{~g} /$ $\mathrm{kg}$ para o versenato de cálcio. ${ }^{29}$

O DMSA, ao contrário do EDTACaNa ${ }_{2}$ por via oral e da D-penicilamina, não aumenta a absorção de chumbo presente do trato gastrintestinal. O DMSA reduz a absorção do chumbo a partir dessa via, corroborando a idéia do uso dessa droga em crianças com níveis sanguíneos elevados, mesmo que ainda estejam expostas em situação ambiental.

O uso de DMSA em crianças intoxicadas por chumbo tem mostrado que existe decréscimo nos níveis de plumbemia durante os dias de tratamento, com retorno gradual logo em seguida a níveis médios num patamar inferior ao pré-tratamento. Um estudo que analisou dados de 39 crianças tratadas mostrou esse padrão de resposta da plumbemia, com manutenção de níveis baixos até cerca de quatro semanas durante o tratamento e re-elevação dos mesmos na $5^{\mathrm{a}}$ ou $6^{\mathrm{a}}$ semanas, com a suspensão do tratamento, mas em níveis médios $10 \mathrm{mcg} / \mathrm{dL}$ abaixo dos níveis pré-tratamento. ${ }^{30}$

Em 1978 foram publicados resultados de dois ensaios clínicos em 18 e 11 adultos, respectivamente, expostos a chumbo ocupacionalmente, usando três doses diferenciadas de DMSA por via oral, por cinco dias, comparando com tratamento padrão com versenato de cálcio $\left(\mathrm{EDTACaNa}_{2}\right)$, mostrando eficácia significativa da droga em reduzir níveis de $\mathrm{PbS}$ durante o tratamento e mantê-los em patamar médio mais baixo após suspensão do tratamento. Houve aumento significativo da excreção de chumbo na urina. ${ }^{31}$ Outros dois trabalhos, relatando resultados de séries de casos de adultos tratados, mostraram boas eficácia e segurança no uso do DMSA em adultos e crianças. ${ }^{32,33}$

Um ensaio clínico com 21 crianças com plumbemias variando entre 31 e $49 \mathrm{mcg} / \mathrm{dL}$ (todas com teste de mobilização de chumbo com dose padrão de EDTACaNa ${ }_{2}$ positivo), e usando três subgrupos de doses diferentes contra grupo controle com tratamento tradicional com versenato de cálcio, mostrou uma redução significativa de chumbo no sangue durante $o$ ciclo de tratamento, bem como o aumento da excreção urinária de chumbo e retorno dos níveis de ALAU e ALA-D. O grupo utilizando DMSA não mostrou depleção de metais essenciais como zinco, magnésio, cobre, ferro e cálcio, ao contrário do grupo tratado com versenato. ${ }^{34}$ Os níveis de plumbúria de 24 horas no grupo usando DMSA foram comparáveis aos do

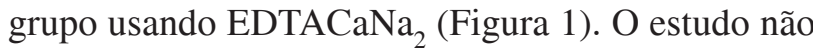
referiu nenhum efeito adverso significativo, acrescentando que em outra série de 50 pacientes de idade entre um e 58 anos, com níveis de plumbemia variando de 31 a $96 \mathrm{mcg} / \mathrm{dL}$, todos tratados com ciclos de cinco dias de DMSA, não houve nenhum efeito colateral que justificasse suspender o agente quelante. Resultados semelhantes quanto à queda de plumbemia foram observados em outro estudo com 18 crianças tratadas. ${ }^{34}$

Um novo estudo clínico foi realizado em 2000 , visando definir a segurança e eficácia do DMSA em crianças com níveis médios de plumbemia entre 25 e $66 \mathrm{mcg} / \mathrm{dL} .{ }^{30}$ Incluiu 59 crianças de idade entre $12 \mathrm{e}$ 65 meses. A média de PbS pós-tratamento caiu para $23 \mathrm{mcg} / \mathrm{dL}$, contra $40 \mathrm{mcg} / \mathrm{dL}$ pré-DMSA. Não se observaram efeitos adversos significativos com o uso do agente por cinco dias. As alterações laboratoriais dignas de nota foram a elevação da fosfatase alcalina em duas crianças, com reversão ao normal após o término do tratamento e, em um paciente, o aumento de eosinófilos circulantes de $1 \%$ para $25 \%$, com queda para $4 \%$ após suspensão do tratamento. $\mathrm{O}$ aumento discreto de transaminases já havia sido observado em testes anteriores. ${ }^{30}$

Diferentemente do versenato de cálcio, o DMSA parece mobilizar chumbo de diversos compartimentos teciduais, como cérebro, rins, fígado e osso trabecular, sem causar redistribuirão que provoque piora nos sintomas ou coloque em risco órgãos nobres como o cérebro e os rins. Alguns estudos mostram que já no primeiro dia de tratamento há excreção de chumbo em quantidade maior que o chumbo contido no compartimento extracelular. ${ }^{29,35}$

Dos estudos clínicos até agora realizados, todos com ciclos de cinco dias, comprovou-se que a dose de $30 \mathrm{mg} / \mathrm{kg} / \mathrm{dia}$ é uma dose segura e eficaz, promovendo decréscimo de plumbemia e aumento na plumbúria. Alguns desses estudos mostraram também que um prolongamento do ciclo por mais 14 dias, na dose de $20 \mathrm{mg} / \mathrm{kg} / \mathrm{dia}$, mostrou-se eficaz na manutenção de níveis de $\mathrm{PbS}$ baixos após o final do tratamento, principalmente em pacientes com grande carga corpórea, como ocorre em exposições crônicas tanto ambientais como ocupacionais. O uso contínuo de DMSA nesses casos, ou seja, em períodos maiores de 19 ou 20 dias, ainda não foi estudado quanto à segurança e eficácia. ${ }^{28}$ 


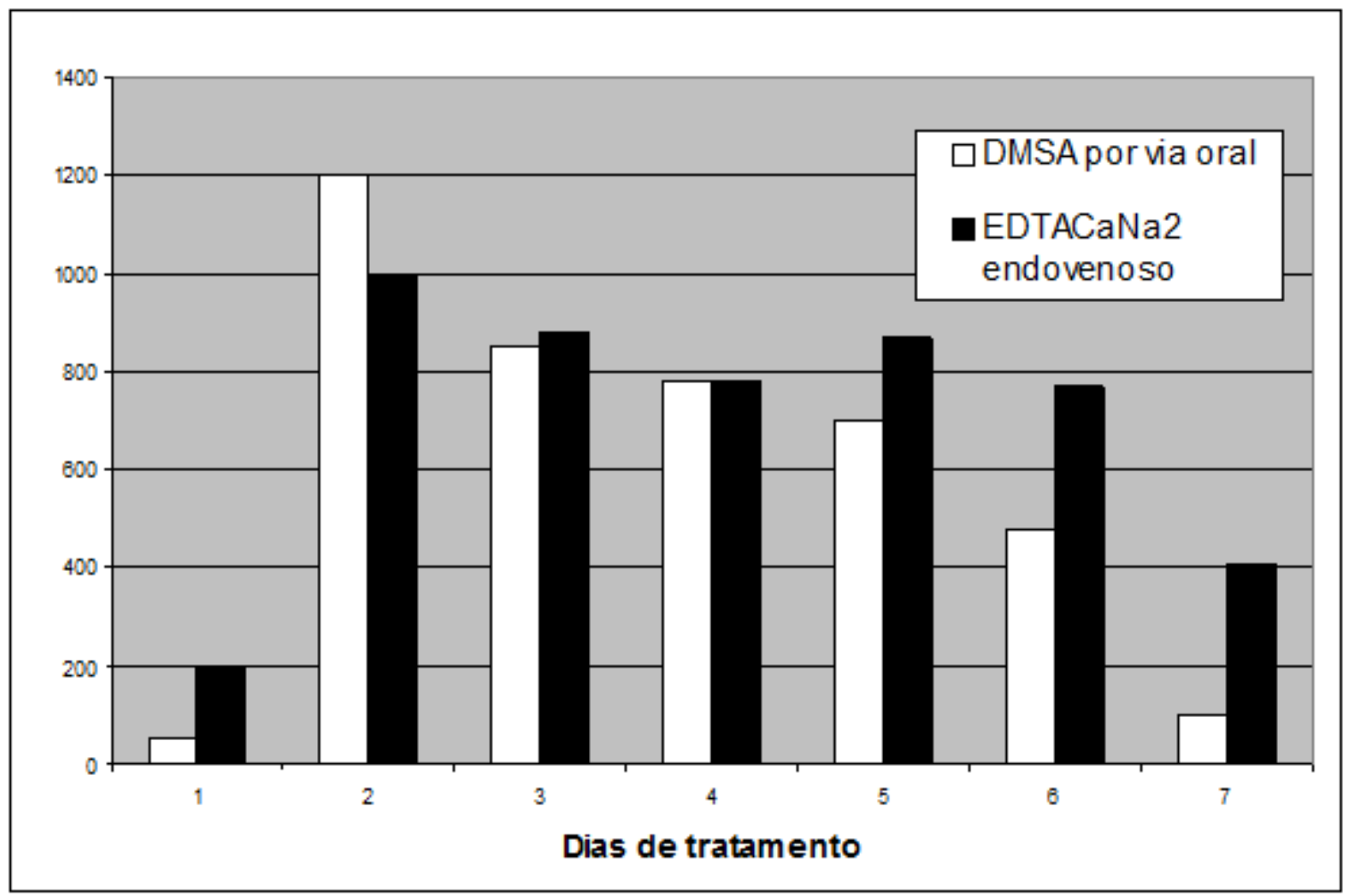

Figura 1. Médias de plumbúria de 24 horas $(\mu \mathrm{g} / \mathrm{g} \mathrm{C})$ em crianças, comparando tratamentos com DMSA por via oral e EDTACaNa2 por via endovenosa. ${ }^{34}$

O DMSA parece ter mais vantagens que o EDTACaNa2 e o BAL no tratamento de crianças intoxicadas por chumbo: é droga menos tóxica, apresentando mínimos efeitos colaterais; é altamente eficaz por via oral, apesar da baixa absorção (máximo de $20 \%$ da dose), ao contrário do versenato de cálcio, que não ultrapassa 5\% de absorção; é mais especifi- ca que o versenato, não causando depleção de metais essenciais como zinco e ferro; não causa hemólise em deficientes de G6PD; e, finalmente, parece não haver contra-indicação ao uso concomitante de ferro suplementar, o que é um dado importante no tratamento de crianças que, em geral, apresentam deficiência de ferro, necessitando reposição.

\section{ABSTRACT}

Diagnosis of lead intoxication must be based basically on signs and symptoms produced by the main affected target organs: brain, hemopoietic system, kidneys, and periphery nervous system. The critical organ is the brain, promoting signs and symptoms of encephalopathy more or less pronounced according to the dose and duration of exposure. Such a symptoms are: headache, memory loss, concentration and attention problems, humor changes, irritability, depression, insomnia, somnolence, stupor, convulsions and coma. Diagnosis can be confirmed by blood and urine lead measurement, or by dosage of any parameter of lead effect on hemoglobin formation, like delta aminulevulinic acid in blood or urine and blood erythrocyte protoporphirin. Treatment is performed using chelating agents. Considering costs and commercial availability Brazilian experience regarding chelating agents for lead intoxications is limited to the use of dimecaprol (BAL), calcium versenate (EDTACaNa2) and D-penicilamine. However, the most efficacious drugs are intravenous EDTACaNa2, and oral dimercapto succinic acid (DMSA). BAL can be used together with EDTACaNa2 in adults when encephalopathic signs are present, or in children using EDTACaNa2 even without CNS signs and symptoms because BAL can pass blood-brain barrier chelating lead in the brain and protecting children against lead mobilization from other tissues to the brain provoked by EDTACaNa2.

Keywords: Lead. Lead Poisoning. Diagnosis. Treatment. 


\section{Referências Bibliográficas}

1. Paoliello MMB, De Capitani EM. Chumbo. In: Azevedo AA, M. CAA, eds. Metais - Gerenciamento da Toxicidade São Paulo: Atheneu-Intertox; 2003:353-98.

2. Dal Molin F, Paoliello MMB, De Capitani EM. A zincoprotoporfirina como indicador biológico na exposição ao chumbo: uma revisão. Rev Bras Toxicol. 2006;19:71-80.

3. Nadig RJ. Treatment of lead poisoning. Jama 1990;263:21812.

4. de Madureira PR, De Capitani EM, Vieira RJ. Lead poisoning after gunshot wound. Sao Paulo Med J. 2000;118:78-80.

5. Somervaille L, Chettle D, Scott M, et al. In vivo tibia lead measurement as an index of cumulative exposure in occupationally exposed subjects. Br J Ind Med. 1988;45:174-81.

6. Somervaille LJ, Nilsson U, Chettle DR, et al. In vivo measurements of bone lead-a comparison of two x-ray fluorescence techniques used at three different bone sites. Phys Med Biol. 1989;34:1833-45.

7. Ahlgren L, Liden K, Mattsson S, Tejning S. X-ray fluorescence analysis of lead in human skeleton in vivo. Scand $J$ Work Environ Health. 1976;2:82-6.

8. Ahlgren L, Haeger-Aronsen B, Mattsson S, Schutz A. In-vivo determination of lead in the skeleton after occupational exposure to lead. Br J Ind Med. 1980;37:109-13.

9. Schutz A, Skerfving S, Christoffersson JO, Ahlgren L, Mattson $S$. Lead in vertebral bone biopsies from active and retired lead workers. Arch Environ Health .1987;42:340-6.

10. CDC. Preventing lead poisoning in young children - a statement by the Centers for Disease Control - Oct 1991: USDHHSPHS-CDCP; 1991.

11. CDC. Screening young children for lead poisoning: guidance for state and local public helath officials. In: Program. U-CCIPP, ed. Atlanta; 1997.

12. Needleman HL, Gunnoe C, Leviton A, et al. Deficits in psychologic and classroom performance of children with elevated dentine lead levels. N Engl J Med.1979;300:689-95.

13. Needleman HL, Schell A, Bellinger D, Leviton A, Allred EN. The long-term effects of exposure to low doses of lead in childhood. An 11-year follow-up report. N Engl J Med. 1990; 322: 83-8.

14. Rogan WJ, Dietrich KN, Ware JH, et al. The effect of chelation therapy with succimer on neuropsychological development in children exposed to lead. N Engl J Med. 2001;344:1421-6.

15. Tong S, Baghurst PA, Sawyer MG, Burns J, McMichael AJ. Declining blood lead levels and changes in cognitive function during childhood: the Port Pirie Cohort Study. Jama. 1998; 280:1915-9.

16. Stokes L, Letz R, Gerr F, et al. Neurotoxicity in young adults 20 years after childhood exposure to lead: the Bunker Hill experience. Occup Environ Med. 1998; 55:507-16.

17. Porru S, Alessio L. The use of chelating agents in occupational lead poisoning. Occup Med. (Lond) 1996; 46:41-8.

18. Markowitz ME, Rosen JF. Need for the lead mobilization test in children with lead poisoning. J Pediatr. 1991;119:305-10.
19. Lee BK, Schwartz BS, Stewart W, Ahn KD. Provocative chelation with DMSA and EDTA: evidence for differential access to lead storage sites. Occup Environ Med. 1995;52:13-9.

20. Markowitz ME, Rosen JF. Assessment of lead stores in children: validation of an 8-hour CaNa2EDTA provocative test. Pediatrics. 1984;104:337-41.

21. Apostoli P, Porru S, Duca P, Ferioli A, Alessio L. Significance and validity of a shortened lead chelation test. J Occup Med. 1990;32:1124-9.

22. De Capitani EM, Paoliello MMB. Diagnóstico e Tratamento das Intoxicações. In: Azevedo AA, M. CAA, eds. Metais - Gerenciamento da Toxicidade. São Paulo: Atheneu-Intertox; 2003: 415-50.

23. Klaassen CD. Heavy metals and heavy-metal antagonists. In: Hardman JG, Limbird LE, eds. Goodman \& Gilman's The Pharmacological Basis of Therapeutis. 9th ed. New York: McGrawHill; 1996:1905.

24. Cardani A, Farina G. Profilassi del saturnismo mediante somministrazione di versene per via intramoscolare. Med Lav. 1970;61:220-6.

25. Sachs HK, Blanksma LA, Murray EF, O'Connell MJ. Ambulatory treatment of lead poisoning: report of 1,155 cases. Pediatrics. 1970;46:389-96.

26. De Capitani EM, de Madureira PR, Moreira Filho DC, et al. Estudo comparativo de tratamento quelante de pacientes intoxicados por chumbo inorgânico com EDTACaNa2 por duas vias de administração. Rev Bras Med Trab. 2004;2:185-90.

27. Cory-Slechta DA, Weiss B, Cox C. Mobilization and redistribution of lead over the course of calcium disodium ethylenediamine tetraacetate chelation therapy. J Pharmacol Exp Ther. 1987;243:804-13.

28. Graziano JH. 2,3-dimercaptosuccinic acid (DMSA, Succimer). In: Goldfrank LR, ed. Goldfrank's Toxicologic Emergencies. 5th ed. Norwalk: Appleton \& Lange; 1994:1045-47.

29. Jorgensen FM. Succimer: the first approved oral lead chelator. Am Fam Physician. 1993;48:1496-502.

30. Chisolm JJ, Jr. Safety and efficacy of meso-2,3dimercaptosuccinic acid (DMSA) in children with elevated blood lead concentrations. J Toxicol Clin Toxicol. 2000; 38:36575.

31. Friedheim E, Graziano JH, Popovac D, Dragovic D, Kaul B. Treatment of lead poisoning by 2,3-dimercaptosuccinic acid. Lancet. 1978;2:1234-6.

32. Grandjean P, Jacobsen IA, Jorgensen PJ. Chronic lead poisoning treated with dimercaptosuccinic acid. Pharmacol Toxicol. 1991;68:266-9.

33. Thomas DJ, Chisolm J, Jr. Lead, zinc and copper decorporation during calcium disodium ethylenediamine tetraacetate treatment of lead-poisoned children. J Pharmacol Exp Ther 1986; 239: 829-35.

34. Graziano JH, Lolacono NJ, Meyer P. Dose-response study of oral 2,3-dimercaptosuccinic acid in children with elevated blood lead concentrations. J Pediatr. 1988;113:751-7.

35. Graziano J, Siris E, Lolacono N, Silverberg S, Turgeon L. 2,3Dimercaptosuccinic acid as an antidote for lead intoxication. Clin Pharmacol Ther. 1985;37:431-8. 